\title{
The Schur Multiplier of a Generalized Baumslag-Solitar Group
}

\author{
DEREK J. S. ROBINSON
}

ABSTRACT - The structure of the Schur multiplier of an arbitrary generalized Baumslag-Solitar group is determined and applications to central extensions are described.

\section{Introduction and Results.}

A generalized Baumslag-Solitar group, or GBS-group, is the fundamental group of a finite connected graph of groups with infinite cyclic vertex and edge groups. In detail let $\Gamma$ be a finite connected graph multiple edges and loops are allowed - with vertex set $V(\Gamma)$ and edge set $E(\Gamma)$. For each edge $e$ we choose endpoints $e^{+}$and $e^{-}$, and hence a direction for the edge. Infinite cyclic groups $\left\langle g_{x}\right\rangle$ and $\left\langle u_{e}\right\rangle$ are assigned to each vertex $x$ and edge $e$. Injective homomorphisms $\left\langle u_{e}\right\rangle \rightarrow\left\langle g_{e^{+}}\right\rangle$and $\left\langle u_{e}\right\rangle \rightarrow\left\langle g_{e^{-}}\right\rangle$are defined by the assignments

$$
u_{e} \mapsto g_{e^{+}}^{\omega^{+}(e)} \text { and } u_{e} \mapsto g_{e^{-}}^{\omega^{-}(e)},
$$

where $\omega^{+}(e), \omega^{-}(e) \in Z^{*}=Z \backslash\{0\}$. Thus we have a weight function

$$
\omega: E(\Gamma) \rightarrow Z^{*} \times Z^{*}
$$

where $\omega(e)=\left(\omega^{-}(e), \omega^{+}(e)\right)$. The weighted graph $(\Gamma, \omega)$ is called a $G B S$ graph.

The GBS-group determined by the weighted graph $(\Gamma, \omega)$ is the fundamental group

$$
G=\pi_{1}(\Gamma, \omega)
$$

(*) Indirizzo dell'A.: Department of Mathematics, University of Illinois at Urbana-Champaign, Urbana, IL 61801, USA.

E-mail: dsrobins@illinois.edu 
To obtain a presentation of $G$ choose a maximal subtree $T$ of $\Gamma$. Then $G$ has generators

$$
g_{x},(x \in V(\Gamma)), \text { and } t_{e},(e \in E(\Gamma) \backslash E(T)),
$$

with defining relations

$$
\left\{\begin{array}{l}
g_{e^{+}}^{\omega^{+}(e)}=g_{e^{-}}^{\omega^{-}}(e), \text { for } e \in E(T), \\
\left(g_{e^{+}}^{\omega^{+}(e)}\right)^{t_{e}}=g_{e^{-}}^{\omega^{-}(e)}, \text { for } e \in E(\Gamma) \backslash E(T) .
\end{array}\right.
$$

It is known that up to isomorphism $G$ is independent of the choice of $T$ : for this and other basic properties of graphs of groups see [1], [3], [10]. If $\Gamma$ consists of a single loop with weight $(n, m)$, then $\pi_{1}(\Gamma, \omega)$ is a Baumslag-Solitar group

$$
B S(m, n)=\left\langle t, g \mid\left(g^{m}\right)^{t}=g^{n}\right\rangle .
$$

It is easy to see that GBS-groups are torsion-free. They are obviously finitely presented, and in fact every finitely generated subgroup of a GBS-group is either free or a GBS-group ([6], 2.7: see also [4], 1.2), so such groups are coherent, i.e., all finitely generated subgroups are finitely presented. By an important result of Kropholler ([7]) the non-cyclic GBS-groups are exactly the finitely generated groups of cohomological dimension 2 which have an infinite cyclic subgroup commensurable with its conjugates. It is therefore natural to enquire about homology and cohomology of GBS-groups in dimensions 1 and 2.

Here we are concerned with integral homology: of course $H_{1}(G) \simeq G_{a b}$, the abelianization, while $H_{2}(G)=M(G)$ is the Schur multiplier of $G$. Our principal result describes the structure of the Schur multiplier of an arbitrary GBS-group.

THEOREm 1. Let $G$ be a generalized Baumslag-Solitar group. Then $M(G)$ is free abelian of rank $r_{0}(G)-1$ where $r_{0}(G)$ is the torsion-free rank of $G_{a b}$.

COROLlary 1. The Euler characteristic of a GBS-group is 0.

This follows since the homology groups of a GBS-group $G$ in dimensions $0,1,2$ have torsion-free ranks $1, r_{0}(G), r_{0}(G)-1$ respectively and the alternating sum of these is zero. 
We remark that associated with any GBS-group there is a complex $K(\Gamma, \omega)$ defined in [4]. It can be shown that the Euler characteristic of this complex is zero and this observation is the basis for a topological - but not necessarily shorter - treatment of Theorem 1 . Details will appear elsewhere ([5]).

\section{T-dependence.}

The structure of $G_{a b}$, and hence $r_{0}(G)$, can be found from the abelian presentation of $G_{a b}$ arising from the standard presentation of the GBSgroup $G$ by the usual method of Smith normal form. However, this is a lengthy process and, as only $r_{0}(G)$ is required in order to compute $M(G)$, it is worthwhile to give a simpler method.

Let $G=\pi_{1}(\Gamma, \omega)$ be a $G B S$-group and let $T$ be the chosen maximal subtree of $\Gamma$. Suppose that $e=\langle x, y\rangle \in E(\Gamma) \backslash E(T)$ where $x \neq y$. Now there is a unique path in the tree $T$ from $x$ to $y$, say $x=x_{0}, x_{1}, \ldots, x_{n}=y$. By reading along this path, we obtain a relation $g_{x}^{p_{1}(e)}=g_{y}^{p_{2}(e)}$ where $p_{1}(e)$ and $p_{2}(e)$ are the respective products of the left and right weight values of the edges in the path from $x$ to $y$. If the vector $\left(\omega^{-}(e), \omega^{+}(e)\right)$ is a rational multiple of $\left(p_{1}(e), p_{2}(e)\right)$, then $e$ is said to be $T$-dependent, and otherwise $e$ is $T$-independent. If $e$ is a loop, then by convention $p_{1}(e)=1=p_{2}(e)$ and $e$ is $T$-dependent precisely when $\omega^{-}(e)=\omega^{+}(e)$.

The definition of $T$-dependence may be restated as follows.

LEMma 1. With the above notation, a non-tree edge $e=\langle x, y\rangle$ of a GBS-graph is T-dependent if and only if

$$
\frac{\omega^{-}(e)}{\omega^{+}(e)}=\frac{p_{1}(e)}{p_{2}(e)} .
$$

If every non-tree edge of a GBS-graph is $T$-dependent, the GBS-graph is said to be tree-dependent. The torsion-free rank of the abelianization of a GBS-group can be computed from the following result.

Theorem 2. Let $G=\pi_{1}(\Gamma, \omega)$ be a generalized Baumslag-Solitar group. Then

$$
r_{0}(G)=|E(\Gamma)|-|V(\Gamma)|+1+\varepsilon(\Gamma, \omega)
$$

where $\varepsilon(\Gamma, \omega)=1$ if $(\Gamma, \omega)$ is tree-dependent and otherwise equals 0 . 
(A variant of this result with a different proof appears in [8], Theorem 1.1). We note that, as a consequence of Theorem $2, r_{0}(G)$ can be found by simply inspecting the graph of the GBS-group $G$. Notice also that $\varepsilon(\Gamma, \omega)$ depends only on the GBS-graph $(\Gamma, \omega)$, not on the choice of maximal subtree. Thus the property of tree-dependence is independent of the maximal subtree selected.

We remark that the invariant $\varepsilon$ is closely related to the centre of a GBSgroup and is an important tool in the theory of GBS-groups: it is the subject of an ongoing investigation.

As is well known, knowledge of the structure of the Schur multiplier of a group allows one to draw conclusions about central extensions by the group. As a consequence of Theorem 1 one can determine when all central extensions by a GBS-group $G$ split, i.e., they are direct products. It is shown in Corollary 4 below that every central extension by a generalized Baumslag-Solitar group $G$ splits if and only if $G_{a b}$ is infinite cyclic.

\section{Proof of Theorem 2 .}

Let $G=\pi_{1}(\Gamma, \omega)$ be a $G B S$-group with $T$ a maximal subtree of $\Gamma$. Then $G$ has an abelian presentation with generators $g_{x}, t_{e}$, where $x \in V(\Gamma), e \in E(\Gamma) \backslash E(T)$, subject to the defining relations $g_{e^{-}}^{\left(\omega^{-}(e)\right.}=g_{e^{+}}^{\omega^{+}(e)}$, $(e \in E(\Gamma))$. Put $G_{0}=\left\langle g_{x} \mid x \in V(\Gamma)\right\rangle$; then $G_{0} \simeq \pi_{1}(T, \omega)$ and $r_{0}\left(G_{0}\right) \leq 1$ since each pair of generators of $G_{0}$ is linearly dependent. Since $G_{0}$ has fewer relations than generators, it is infinite and $r_{0}\left(G_{0}\right)=1$. Of course, the stable elements $t_{e}$, are linearly independent modulo the torsion subgroup of $G_{a b}$. Therefore

$$
r_{0}(G)=|E(\Gamma) \backslash E(T)|+\varepsilon,
$$

where $\varepsilon=1$ if each vertex generator has infinite order modulo $G^{\prime}$ and otherwise $\varepsilon=0$. If some non-tree edge $e$ is $T$-independent, then, in the notation of Lemma 1 , the relations $g_{e^{-}}^{\omega^{-}(e)}=g_{e^{+}}^{\omega^{+}(e)}$ and $g_{e^{-}}^{p_{1}(e)}=g_{e^{+}}^{p_{2}(e)}$ are independent, which forces each vertex generator to have finite order modulo $G^{\prime}$; hence $\varepsilon=0$. On the other hand, if all such edges are $T$-dependent, i.e., $(\Gamma, \omega)$ is tree-dependent, then all vertex generators have infinite order and $\varepsilon=1$. Since

$$
|E(\Gamma) \backslash E(T)|=|E(\Gamma)|-(|V(\Gamma)|-1)=|E(\Gamma)|-|V(\Gamma)|+1,
$$

the result follows on setting $\varepsilon(\Gamma, \omega)=\varepsilon$. 


\section{Proof of Theorem 1.}

Let $G=\pi_{1}(\Gamma, \omega)$ be a $G B S$-group with $T$ a maximal subtree of $\Gamma$. We recall the following inequality, which is valid for any finitely presented group $H$ with $n$ generators and $r$ relators:

$$
n-r \leq r_{0}(H)-d(M(H)),
$$

where $d(X)$ is the minimal number of generators of a group $X$, (see, for example, [9], p.550). In the present situation we have $n=|V(\Gamma)|+$ $|E(\Gamma) \backslash E(T)|$ and $r=|E(\Gamma)|$, so $n-r=1$. Thus $d(M(G)) \leq r_{0}(G)-1$ and it suffices to prove that $r_{0}(M(G)) \geq r_{0}(G)-1$. The proof is by induction on $|E(\Gamma)|$, which may be assumed positive.

(i) We can assume that $r_{0}(G)>1$, so that $\Gamma$ is not a tree.

For if $r_{0}(G)=1$, then $d(M(G))=0$. Note that if $\Gamma$ is a tree, then $r_{0}(G)=1$ since each pair of vertex generators is linearly independent.

(ii) Case: $\Gamma$ has a single non-tree edge.

Let $e=\langle x, y\rangle$ be the edge which is not in $T$. Now $r_{0}(G) \leq 2$ by Theorem 2 , so $r_{0}(G)=2$ and $\varepsilon(\Gamma, \omega)=1$; thus $e$ must be $T$-dependent. Apply the five-term homology sequence for the exact sequence $G^{\prime} \succ G \rightarrow G_{a b}$ to get

$$
M(G) \rightarrow M\left(G_{a b}\right) \rightarrow G^{\prime} /\left[G^{\prime}, G\right] \rightarrow G_{a b} \rightarrow G_{a b} \rightarrow 1 .
$$

Note that $r_{0}\left(M\left(G_{a b}\right)\right)=1$ since $M\left(G_{a b}\right) \simeq G_{a b} \wedge G_{a b}$.

We claim that $G^{\prime} /\left[G^{\prime}, G\right]$ is finite. To see this write $t=t_{e}$ and let $\omega(e)=(h, k)$, so that $\left(g_{y}^{k}\right)^{t}=g_{x}^{h}$. Also $\left\langle g_{x}\right\rangle \cap\left\langle g_{y}\right\rangle=\left\langle g_{x}^{m}=g_{y}^{n}\right\rangle$ where $m, n \in Z^{*}$. By $T$-dependence $(h, k)$ is a rational multiple of $(m, n)$, say $i h=j m$ and $i k=j n$, with $i, j \in Z^{*}$. Then

$$
\left[g_{y}, t\right]^{i k} \equiv\left[g_{y}^{i k}, t\right] \equiv g_{y}^{-i k} g_{x}^{i h} \equiv g_{y}^{-j n} g_{x}^{j m} \equiv 1 \bmod \left[G^{\prime}, G\right] .
$$

Next for any vertex generator $g_{z}$ we have $g_{z}^{r}=g_{y}^{s}$ for some $r, s \in Z^{*}$, and hence

$$
\left[g_{z}, t\right]^{r} \equiv\left[g_{y}^{s}, t\right] \equiv\left[g_{y}, t\right]^{s} \bmod \left[G^{\prime}, G\right] .
$$

Finally, $\left[g_{u}, g_{v}\right]\left[G^{\prime}, G\right]$ has finite order for any vertex generators $g_{u}, g_{v}$. It follows that $G^{\prime} /\left[G^{\prime}, G\right]$ is periodic, so it is finite.

Returning to the exact homology sequence above, we conclude that $M(G)$ must be infinite, so that $r_{0}(M(G)) \geq 1=r_{0}(G)-1$, as required. 
(iii) From now on we assume that $\Gamma$ has at least two non-tree edges.

Let $e=\langle x, y\rangle$ be one of the non-tree edges and let the unique path in $T$ from $x$ to $y$ be

$$
\left\langle x_{1}, x_{2}\right\rangle,\left\langle x_{2}, x_{3}\right\rangle, \ldots,\left\langle x_{k-1}, x_{k}\right\rangle
$$

where $x=x_{1}$ and $y=x_{k}$. Define subgroups $G_{1}=\left\langle t_{e}, g_{x_{1}}, \ldots, g_{x_{k}}\right\rangle$ and

$$
G_{2}=\left\langle t_{f}, g_{x} \mid x \in V(\Gamma), f \in E(\Gamma) \backslash E(T), f \neq e\right\rangle .
$$

Then $G_{i}=\pi_{1}\left(\Gamma_{i}, \omega\right), i=1,2$, where $\Gamma_{1}, \Gamma_{2}$ are subgraphs of $\Gamma$ with $V\left(\Gamma_{1}\right)=\left\{x_{1}, \ldots, x_{k}\right\}, V\left(\Gamma_{2}\right)=V(\Gamma)$ and respective edge sets $\left\{e,\left\langle x_{j}, x_{j+1}\right\rangle \mid j=1,2, \ldots, k-1\right\}$ and $E(\Gamma) \backslash\{e\}$, with restricted weight functions. Furthermore

$$
G=G_{1} *_{U} G_{2}
$$

where $U=\left\langle g_{x_{1}}, g_{x_{2}}, \ldots, g_{x_{k}}\right\rangle$. Since $U \simeq \pi_{1}\left(T_{0}, \omega\right)$, with $T_{0}$ the path $\left\langle x_{1}, x_{2}\right\rangle,\left\langle x_{2}, x_{3}\right\rangle, \ldots,\left\langle x_{k-1}, x_{k}\right\rangle$, we have $r_{0}(U)=1$ and $M(U)=0$ by (i).

Next we form the Mayer-Vietoris sequence for the generalized free product $G=G_{1} *_{U} G_{2}$, ([2], p. 51),

$$
0=M(U) \rightarrow M\left(G_{1}\right) \oplus M\left(G_{2}\right) \rightarrow M(G) \rightarrow U_{a b} \rightarrow
$$

$$
\left(G_{1}\right)_{a b} \oplus\left(G_{2}\right)_{a b} \rightarrow G_{a b} \rightarrow 1 .
$$

At this point we must distinguish two cases.

(iv) Case: the graph $\Gamma$ has a non-tree edge e which is T-dependent.

Apply the Mayer-Vietoris sequence above for the edge $e$. Since $\Gamma_{1}$ has just one non-tree edge $e$ and it is $T$-dependent in $\Gamma_{1}$, we conclude that $r_{0}\left(G_{1}\right)=2$ and $M\left(G_{1}\right) \simeq Z_{1}$ by (ii). Also $U G_{1}^{\prime} / G_{1}^{\prime}$ is infinite, so the image of $\left(G_{1}\right)_{a b}$ in the exact sequence $(*)$ has infinite projection into $(G)_{a b}$. Therefore

$$
r_{0}(G) \leq r_{0}\left(G_{1}\right)+r_{0}\left(G_{2}\right)-1=r_{0}\left(G_{2}\right)+1
$$

and $r_{0}\left(G_{2}\right) \geq r_{0}(G)-1$. By induction on $|E(\Gamma)|$ the result is true for $G_{2}$, so we have

$$
r_{0}(M(G)) \geq r_{0}\left(M\left(G_{1}\right) \oplus M\left(G_{2}\right)\right) \geq 1+\left(r_{0}(G)-2\right)=r_{0}(G)-1,
$$

as required.

We are now left with the situation:

(v) Case: all non-tree edges in $\Gamma$ are $T$-independent.

Choose any non-tree edge $e$ and apply the sequence $(*)$ in (iii) for this edge. Since $e$ is $T$-independent, $r_{0}\left(G_{1}\right)=1$ and $M\left(G_{1}\right)=0$. Also $U G_{1}^{\prime} / G_{1}^{\prime}$ is finite because $e$ is $T$-independent. By (iii) there is non-tree edge $f \neq e$ and 
$\bar{t}=t_{f} \in G_{2}$. Since $f$ is $T$-independent, $r_{0}(\langle\bar{t}, U\rangle)=1$ and also $U G_{2}^{\prime} / G_{2}^{\prime}$ is finite. Consequently the image of $U_{a b}$ in $\left(G_{1}\right)_{a b} \oplus\left(G_{1}\right)_{a b}$ is finite. Since $U_{a b}$ is infinite, it follows from the sequence $(*)$ that the cokernel of the map $M\left(G_{1}\right) \oplus M\left(G_{2}\right) \rightarrow M(G)$ is infinite.

By induction hypothesis the result holds for $G_{2}$, so we conclude that

$$
r_{0}(M(G)) \geq r_{0}\left(M\left(G_{1}\right)\right)+r_{0}\left(M\left(G_{2}\right)\right)+1=\left(r_{0}\left(G_{2}\right)-1\right)+1=r_{0}\left(G_{2}\right),
$$

since $M\left(G_{1}\right)=0$. Finally, the image of $U_{a b}$ in the sequence (*) being finite, we obtain

$$
r_{0}(G)=r_{0}\left(\left(G_{1}\right)_{a b} \oplus\left(G_{2}\right)_{a b}\right)=r_{0}\left(G_{1}\right)+r_{0}\left(G_{2}\right)=1+r_{0}\left(G_{2}\right) .
$$

Hence $r_{0}\left(G_{2}\right)=r_{0}(G)-1$ and $r_{0}(M(G)) \geq r_{0}(G)-1$, as required.

COROLLARY 2. The GBS-group $\pi_{1}(\Gamma, \omega)$ has trivial Schur multiplier if and only if $\Gamma$ is either a tree or else a tree with one further edge and $\Gamma$ is not tree-dependent.

Proof. By the theorem $M(G)=0$ if and only if $r_{0}(G)=1$. This condition requires there to be at most one non-tree edge and by Theorem 2 it must be $T$-independent.

\section{Corollary 3. Every GBS-group has deficiency 1.}

Proof. Recall that the deficiency $\operatorname{def}(G)$ of group $G$ is equal to $\sup \{n-r\}$ where $n$ and $r$ are the respective numbers of generators and relations in an arbitrary finite presentation. If $G$ is a GBS-group, then $1 \leq \operatorname{def}(G) \leq r_{0}(G)-d(M(G))=1$.

ExAmPLE. Consider the GBS-group $G$ arising from the following GBS-graph,

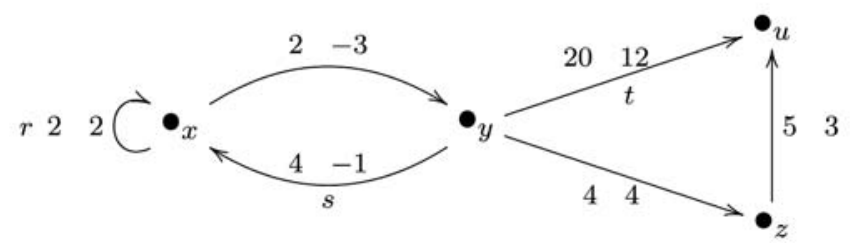

where the maximal subtree chosen is the path $x, y, z, u$ and the stable elements are $r, s, t$ as indicated. Then $G$ has a presentation with generators

$$
r, s, t, g_{x}, g_{y}, g_{z}, g_{u}
$$


and relations

$$
\left(g_{x}^{2}\right)^{r}=g_{x}^{2}, g_{x}^{2}=g_{y}^{-3}, g_{y}^{4}=g_{z}^{4}, g_{z}^{5}=g_{u}^{3},\left(g_{u}^{12}\right)^{t}=g_{y}^{20},\left(g_{x}^{4}\right)^{s}=g_{y}^{-1} .
$$

All non-tree edges with the exception of $\langle y, x\rangle$ are $T$-dependent. Therefore $(\Gamma, \omega)$ is not tree-dependent, $\varepsilon(\Gamma, \omega)=0$ and $r_{0}(G)=$ $|E(\Gamma)|-|V(\Gamma)|+1=3$. Thus $M(G) \simeq Z_{1} \oplus Z$.

\section{Applications to Central Extensions.}

We will now apply our results to yield information about central extensions by GBS-groups. Let $G$ be a $G B S$-group and $C$ an abelian group regarded as a trivial $G$-module. Denote by $F$ the periodic subgroup of $G_{a b}$; thus $G_{a b} \simeq F \oplus Z^{r_{0}(G)}$ where $F$ is finite. By the Universal Coefficients Theorem

$$
H^{2}(G, C) \simeq \operatorname{Ext}\left(G_{a b}, C\right) \oplus \operatorname{Hom}(M(G), C) \simeq \operatorname{Ext}(F, C) \oplus \operatorname{Dr} C^{r_{0}(G)-1}
$$

First we determine when all central extensions of $C$ by $G$ are direct products, i.e., when $H^{2}(G, C)=0$.

THEOREM 3. Let $G$ be a generalized Baumslag-Solitar group and let $C \neq 1$ be an abelian group regarded as a trivial G-module. Then $H^{2}(G, C)=0$ if and only if $r_{0}(G)=1$ and $C$ is divisible by all primes $p \in \pi\left(G_{a b}\right)$.

Proof. With the notation used above, $H^{2}(G, C)=0$ if and only if $\operatorname{Ext}(F, C)=0$ and $r_{0}(G)=1$. Since $F$ is finite and $\operatorname{Ext}\left(Z_{n}, C\right) \simeq C / C^{n}$, it follows that $\operatorname{Ext}(F, C)=0$ if and only if $C=C^{p}$ for all $p \in \pi\left(G_{a b}\right)$. (For the elementary properties of Ext used here see [9], 7.2).

COROLlary 4. The following conditions on a generalized BaumslagSolitar group $G$ are equivalent

(i) $H^{2}(G, Z)=0$;

(ii) $G_{a b} \simeq Z$;

(iii) $H^{2}(G, C)=0$ for all abelian groups $C$.

Proof. Clearly condition (i) implies that $\pi\left(G_{a b}\right)$ is empty and so (ii) holds. Also (ii) implies (iii), while trivially (iii) implies (i). 
For example, if $G=B S(m, n)$, then $G_{a b} \simeq Z \oplus Z_{|m-n|}$, so that $G$ has the property of Corollary 4 if and only if $|m-n|=1$.

There are corresponding results for homology, which can be proved in an analogous way by using the Universal Coefficients Theorem for homology,

$$
H_{2}(G, C) \simeq \operatorname{Tor}\left(G_{a b}, C\right) \oplus(M(G) \otimes C),
$$

and elementary properties of Tor, (see [9], 7.1).

THEOREm 4. Let $G$ be a generalized Baumslag-Solitar group and let $C \neq 1$ an abelian group regarded as a trivial G-module. Then $H_{2}(G, C)=0$ if and only if $r_{0}(G)=1$ and $C_{p}=1$ for all primes $p \in \pi\left(G_{a b}\right)$.

COROLlary 5. The following conditions on a generalized BaumslagSolitar group $G$ are equivalent:

(i) $\mathrm{H}_{2}(G, \mathrm{Q} / \mathrm{Z})=0$;

(ii) $G_{a b} \simeq Z$;

(iii) $H_{2}(G, C)=0$ for all abelian groups $C$.

\section{REFERENCES}

[1] O. BoGopolski, Introduction to Group Theory, European Math. Soc., Zürich, 2008.

[2] K. S. BRown, Cohomology of Groups, Graduate Texts, vol. 87, Springer, New York, 1982.

[3] D. E. Cohen, Combinatorial Group Theory: a Topological Approach, London Math. Soc., Student Texts, vol. 14, Cambridge, 1989.

[4] A. L. Delgado - D. J. S. Robinson - M. Timm, Generalized Baumslag-Solitar groups and geometric homomorphisms, J. Pure Appl. Algebra, 215 (2011), pp. 398-410.

[5] A. L. Delgado - D. J. S. Robinson - M. Timm, The topology of generalized Baumslag-Solitar groups, preprint.

[6] M. Forester, On uniqueness of JSG decompositions of finitely generated groups, Comment. Math. Helv., 78 (2003), pp. 740-741.

[7] P. H. KrophoLLER, Baumslag-Solitar groups and some other groups of cohomological dimension two, Comment. Math. Helv., 65 (4) (1990), pp. 547-558.

[8] G. LeVITT, On the automorphism group of generalized Baumslag-Solitar groups, Geom. Topol., 11 (2007), pp. 473-515.

[9] J. J. Rotman, An Introduction to Homological Algebra, 2nd ed., Springer, New York, 2008.

[10] J.-P. SerRe, Trees, translated from the French by J. Stillwell, Springer, Berlin, 1960.

Manoscritto pervenuto in redazione il 7 ottobre 2010. 
